\title{
2
}

\section{CODIFIED RELIABILITY OF STRUCTURES}

\author{
O. Ditlevsen, Department of Structural Engineering, Technical University of Denmark, \\ Build. 118, DK 2800 Lyngby, Denmark
}

\begin{abstract}
For the practical applications of probabilistic reliability methods it is important to make decisions about the target reliability level. Presently calibration to existing design practice seems to be the only practicable and politically reasonable solution to this decision problem. However, several difficulties of ambiguity and definition show up when attempting to make the transition from a given authorized partial safety factor code to a superior probabilistic code. For any chosen probabilistic code format there is a considerable variation of the reliability level over the set of structures defined by the partial safety factor code. Thus there is a problem about which of these levels to choose as target level. Moreover, if two different probabilistic code formats are considered, then a constant reliability level in the one code does not go together with a constant reliability level in the other code. The last problem must be accepted as the state of the matter, and it seems that it can only be solved pragmatically by standardizing a specific code format as reference format for constant reliability.

By an example this paper illustrates that a presently valid partial safety factor code imposes a quite considerable variation of the reliability measure as defined by a specific probabilistic code format. Decision theoretical principles are applied to get guidance about which of these different reliability levels of existing practice to choose as target reliability level. Moreover it is shown that the chosen probabilistic code format not only has strong influence on the formal reliability measure but also on the formal cost of failure to be associated if a design made to the target reliability level is considered to be optimal. In fact, the formal cost of failure can be different by several orders of size for two different by and large equally justifiable probabilistic code formats. Thus the consequence is that a decision theoretical code format formulated as an extension of a probabilistic code format must specify formal values to be used as costs of failure.

A principle of prudency is suggested for guiding the choice of the reference probabilistic code format for constant reliability. To the author's opinion there is an urgent need for establishing a standard probabilistic reliability code. This paper presents some considerations that may be debatable but nevertheless point at a systematic way to choose such a code.
\end{abstract}

\section{Introduction}

It may prevent confusion about the meaning of the concept of limit state, if the concept is formally introduced without a specific physical interpretation. Then the limit state concept is just a mathematical criterion that categorizes any value set of the relevant structural variables (load-, strength-, geometrical variables) into one of two categories, the "desirable" category ("safe" set) and the "adverse" category ("failure" set). The word failure then means "failure of satisfying the limit state criterion" rather than a failure in the sense of a dramatic physical event.

The purpose of introducing the limit state is to represent any chosen kind of less desirable physical behavior (adverse behavior) in a sufficiently idealized way for setting up a set of practically operational rules that ensure that such adverse behavior rarely occurs, given that the rules are followed. 
Codified probabilistic reliability theory contains a definition of a measure of rareness of violation of any considered limit state. For example, this measure may be defined as the reliability index $\beta$ with respect to the limit state (technically: $\beta=-\Phi^{-1}\left(p_{f}\right), \Phi(\cdot)=$ standardized normal distribution function, $p_{f}=$ probability of adverse behavior calculated by use of codified probability distribution types for the basic variables).

The problem is, however, to decide what "rarely" means. It seems obvious that the reliability index corresponding to a given limit state should depend on the physical consequence (and thus the socio-economical consequence) of the occurrence of the adverse behavior. This leads directly to the rationale of cost-benefit analysis (decision theory). While such an analysis in a rational way can lead to a determination of the relative variation of the reliability index over a set of different limit states, there remains the problem of choosing the absolute reliability level for the structure as it will be physically represented when built. Since the costs of consequences of structural damage or collapse embrace intangible socio-economic costs such as injury or loss of human lives and possibly irremediable damages on the environment, there seems to be no other way than to establish some fix-points in the most frequent existing practice and calibrate the reliability level to these fix-points.

This point of view, however, presents a problem of what is most frequent existing practice. An illustrative example concerns the design of reinforced concrete structures. It is current practice in some countries and among some consulting engineers that the ultimate (the most adverse) limit state is defined in terms of load effects and stresses obtained from the theory of elasticity. In other countries it has become practice to define the ultimate limit state as corresponding to a plasticity-theoretical collapse. In spite of these differences of defining the ultimate limit state, existing practice has through codification of a fixed set of partial safety factors by and large assigned the same formal reliability level to the two different types of limit states. However, except if the elasticity theoretical formulas have been corrected on the basis of carrying capacity experiments to represent the collapse situation, the consequences of violating the "elastic" ultimate limit state are in several important examples less adverse with respect to the physical consequences than the consequences of collapse as idealized by the "plastic" ultimate limit state. This means that reinforced concrete designs made according to the elastic ultimate limit state have larger reliability with respect to real physical collapse than designs made according to the plastic ultimate limit state.

For code calibration purposes the problem is now which of the two reliability levels with respect to collapse should be codified in a probabilistic code. It is by certain parties claimed that only the current design practice based on the elastic ultimate limit state provides sufficient structural reliability; if this is so, then several existing reinforced concrete structures are underdesigned. On the other hand, since such structures designed according to the (effectivity factor adapted) plasticity theory with usual partial safety factors have been accepted by the society (the authorities), and since such existing concrete structures show no particular indications of weaknesses, it can be claimed that their general reliability level is sufficiently high. There is also a stronger argument for accepting the plastic ultimate limit state as the ultimate limit state for which the codified reliabil- 
ity level should be valid. This stronger argument is that the designs made according to the elastic ultimate limit state have nonuniform reliability with respect to similar consequences. In some cases of current practice the elastic and the plastic ultimate limit states are made practically coincident (by use of empirical correction factors, e.g. in the case of bending failures of statically determinate shallow beams); in other cases there are very large differences (for example for shear walls). It is, however, a well established experience that the violation of the plastic ultimate limit state implies that a situation close to structural collapse occurs. By and large this experience is valid uniformly over the different types of reinforced concrete structures (plates and shells excepted, perhaps). The occurrence of the adverse event defined by the elastic ultimate limit state may or may not indicate such a near collapse situation.

Let us make the supposition that there in a given country (or union of cooperating countries) is a decision maker (a committee, say) that possesses superior authority with respect to deciding what is optimal structural design. This decision maker is imagined to be asked to point out a specific structure type (structural element type, say) that will get optimal dimensions if it is designed to the limit according to a given partial safety factor code and a specific ultimate limit state model both specified by the authority itself. This means that the superior authority by answering this question implicitly evaluates the intangible socio-economic cost of adverse behavior of the considered structure type such that any stronger or weaker design will have a larger total cost than that of the optimal design. In fact, if the decision theory of von Neumann and Morgenstern [9] is accepted as a valid principle of decision making the evaluation of the socio-economic costs of the different possible consequences of the decision is precisely the task of the decision maker. The minimization of the total expected cost then leads to the optimal decision. With the optimal decision given the intangible cost of adverse behavior can therefore be backcalculated under the adoption of a specific probabilistic code. In this way having calibrated the intangible cost of the design decision problem to the authoritative declaration of optimality, the future design decisions for the considered structure type can be based on rational decision theory implemented within the given probabilistic code.

Even though the superior authority might admit that its partial safety factor code may be too simplified to have the potential of giving optimal designs for all the structure types for which the code is used, there should be at least one structure type within the domain of the code that by the authority is considered to be optimally designed. Otherwise the authority is not self-consistent and as the proper authority it should therefore change the value settings of the code [1]. Therefore the outlined strategy for setting up a probabilistic code that can be approved by the superior authority seems reasonable. However, more detailed analysis reveals several problems that require authoritative decisions.

The first problem to be illustrated in the next section is that the authority is not asked which specific structure of the considered type is the optimal structure. Of course, such a question can be asked, but as it will become evident in the following it is not necessary and hardly wise to require such a specific and difficult question to be directly answered by the authority. By only declaring optimality for the specified structure type class as such it turns out that for a given probabilistic code there is a considerable uncertainty 
about the value of the optimal reliability index. It will be shown that this problem can be solved in a rational way by a decision theoretical argument.

If the authority declares that two or more classes of structure types within the domain of the partial safety factor code possessoptimality then the corresponding reliability indices and intangible costs of adverse behavior can be calculated for each structure type by use of the given probabilistic code. Of course, it is not necessarily so that these costs should be the same for all structure classes. However, it may be a surprise to the authority if the ordering of the structure classes according to increasing cost of adverse behavior turns out not to be consistent with the ordering obtained from a realistic engineering evaluation of the consequences of the adverse behavior. Such experience should force the authority to do a reexamination of its decisions about optimality. Having consistence in the ordering it may also become clear to the authority that the calculated costs of adverse behavior should be adjusted relative to each other to better reflect the engineering judgement of the consequences of the adverse behavior. Of course, at this state the authority should totally abandone the original partial safety factor code and replace it by the probabilistic code as the superior reference for codified structural reliability. Future simplified codes of type as partial safety factor formats or other simplified code formats should then be calibrated to this superior probabilistic reference code.

The philosophical problems of establishing a standardized probabilistic code are not over by the above strategy. There remains the problem of which probabilistic code format to standardize, that is, which types of probability distributions to assign to the specified list of basic variables of the code. The often discussed tail sensitivity problem cannot be ruled out solely by supporting the choice on empirical evidence and physical modeling. By a simple example the ambiguity problem is shown to be serious. Calculations made by Sørensen and Enevoldsen [2] may be taken as an indication that there may even be a problem of whether two different equally possible probabilistic code formats define the same ordering with respect to the reliability index. To come to a decision about which format to choose among a finite set of probabilistic code formats a prudency principle is suggested that ensures as limited deviation from the authoritatively instituted partial safety factor design practice as possible.

All these different aspects are discussed in the following. The paper advocates a rational and operational procedure to transfer present codified practice into a more structured and rational probabilistic code. An attempt to formulate an example of how such a code could look like (without any calibrations to existing codes) is given by Ditlevsen and Madsen [6] as a part of the activity within the Joint Committee on Structural Safety (JCSS).

As probabilistic reliability methods gain increasing interest in engineering practice as judged from the growing community of reliability engineers and from the increasing number of conferences on the subject, it becomes increasingly more urgent to reach general concensus about the standardization on a specific probabilistic code format.

This paper is meant as a debate paper on this topic. The first draft of the paper was presented to JCSS for discussion leading to extensive revisions. It is emphasized that the statements of this paper are solely expressing the opinion of the author and they cannot 
be taken as approved by JCSS.

If should finally be mentioned that several code calibration applications of ad hoc probabilistic reliability models without reference to a standardized probabilistic code format have been reported in the literature $[1,3,4,5]$.

\section{Illustrative example}

As an illustration let us make the supposition that the superior authority points at the structure type class consisting of the tubular joints of an offshore structure as being optimal if the joints are designed according to the Danish offshore code (DS 449) [7] as explained in the following.

For a standard type plane tubular joint in an offshore structure the limit state with respect to failure of the joint is assumed to be modeled by an empirically based equation of the form

$1-\frac{P}{P_{u}}-\left(\frac{M}{M_{u}}\right)^{\alpha_{1}}-\left(\frac{N}{N_{u}}\right)^{\alpha_{2}}=0$

in which $P$ is an internal normal force, $M$ is an internal moment in the plane of the joint, and $N$ is an internal moment orthogonal to the plane. The corresponding strength variables are the random variables $P_{u}, M_{u}$, and $N_{u}$, respectively. Further details about the geometry and mechanical behavior of the joint are not needed in this example. For each joint there is an influence matrix $\boldsymbol{C}$ such that the internal forces of the joint are obtained as the linear function $[P M N]^{\prime}=C[G Q S V W]^{\prime}$ of the five random load variables $G$ (self weight), $Q$ (operational load), $S$ (snow and ice load), $V$ (wind load), and $W$ (wave and current load).

All eight random variables are assumed to be mutually independent even though this assumption may not be fully realistic. In particular the three strength variables could be mutually dependent as also the nature loads $S, V$, and $W$ could be dependent. However, for the purpose of this illustration these possible dependencies are not essential. Moreover the problem is simplified by assuming that the exponents $\alpha_{1}$, and $\alpha_{2}$ are deterministic: $\alpha_{1}=2.1, \alpha_{2}=1.2$.

The probabilistic code is defined by specifying that the three resistance random variables $P_{u}, M_{u}, N_{u}$ have lognormal distributions of common expectation $\theta$ and coefficients of variation $0.20,0.25,0.25$, respectively, and that the load variables $G, Q, S, V, W$ have normal distributions of common mean 1 (with a suitably physical unit) and coefficients of variation $0.05,0.15,0.20,0.25,0.30$, respectively. The common expectation $\theta$ of the strength variables is taken as the design variable ideally to be determined such that the joint gets a prespecified reliability with respect to the limit state (1).

We now make the supposition that the joint is chosen at random among all the joints of a large offshore structure. This means that the influence matrix $\boldsymbol{C}$ is an outcome of a random matrix. It is assumed that the value of the design variable $\theta$ for the chosen joint is obtained according to the Danish offshore code (DS 449) (the format of which is similar 
to the general format of the Euro-codes) [7]. Thus $\theta$ is determined as the largest of the five values obtained according to the five design load combinations

$$
\left[\begin{array}{c}
p_{d 1} \cdots p_{d 5} \\
m_{d 1} \cdots m_{d 5} \\
n_{d 1} \cdots n_{d 5}
\end{array}\right]=\boldsymbol{C}\left[\begin{array}{ccccc}
\gamma_{g} g_{k} & \gamma_{g} g_{k} & \gamma_{g} g_{k} & \gamma_{g} g_{k} & 1.15 g_{k} \\
\gamma_{f} q_{k} & \psi_{q} q_{k} & \psi_{q} q_{k} & \psi_{q} q_{k} & 0 \\
\psi_{s} s_{k} & \gamma_{f} s_{k} & \psi_{s} s_{k} & \psi_{s} s_{k} & 0 \\
\psi_{v} v_{k} & \psi_{v} v_{k} & \gamma_{f} v_{k} & \psi_{v} v_{k} & 0 \\
\psi_{w} w_{k} & \psi_{w} w_{k} & \psi_{w} w_{k} & \gamma_{f} w_{k} & 0
\end{array}\right]
$$

substituted together with the design values $p_{u d}=p_{u k} / \gamma_{m}, m_{u d}=m_{u k} / \gamma_{m}, n_{u d}=n_{u k} / \gamma_{m}$ of the resistance variables into the limit-state equation (1). The characteristic values $p_{u k}, m_{u k}, n_{u k}$ are defined as the $5 \%$-fractiles of the distributions of $P_{u}, M_{u}, N_{u}$, respectively. For the assumed data of the probabilistic code these characteristic values are $\left[\begin{array}{lll}p_{u k} & m_{u k} & n_{u k}\end{array}\right]=\left[\begin{array}{lll}0.708 & 0.647 & 0.647\end{array}\right] \theta$. The material strength partial safety factor is put to $\gamma_{m}=1.2$. The characteristic values $q_{k}, s_{k}, v_{k}, w_{k}$ of the loads $Q, S, V, W$ are defined as the $98 \%$-fractile in the distribution of the yearly extreme, while the characteristic value $g_{k}$ of the self-weight $G$ is defined as the $50 \%$ fractile. Thus $\left[\begin{array}{lllll}g_{k} & q_{k} & s_{k} & v_{k} & w_{k}\end{array}\right]=$ [1.000 1.308 1.4111 .514 1.616]. The last matrix in (2) contains the design values obtained from the characteristic values by application of the partial coefficients $\gamma_{g}$ and $\gamma_{f}$ on selfweight and variable load, respectively, and the load combination factors $\psi_{q}, \psi_{s}, \psi_{v}, \psi_{w}$. These factors are put to $\gamma_{g}=1.0, \gamma_{f}=1.3, \psi_{q}=1.0, \psi_{s}=\psi_{v}=\psi_{w}=0.5$.

In order to assess how much the reliability varies among the joints of the considered offshore structure given that all joints are designed according to the partial safety factor code defined here, it is assumed as an example that the 15 influence numbers in $\boldsymbol{C}$ are mutually independent random variables that are uniformly distributed between 0 and 1. Bjerager [8] considered this example and simulated 1000 outcomes of $\boldsymbol{C}$, and for each of these the design parameter value $\theta$ and thereafter the geometric reliability index $\beta$ (Hasofer-Lind) were calculated. The obtained sample of 1000 values of $\beta$ turned out to be reasonably well described by a normal distribution of mean $\mu \simeq 4.40$ and a standard deviation $\sigma \simeq 0.30$. Thus there is a considerable variability of the reliability index resulting from constant partial safety factor design. This variability is comparable in size with the difference between two neighbour safety classes as they are defined by The Nordic Committee on Building Regulations [4]. The difference between high safety class and normal safety class is expressed by a difference in the $\beta$-level of 0.5 , Fig. 1.

On the basis of this result it is seen that the probability $p_{f}$ of occurrence of failure within one year and the corresponding reliability index $\beta_{\text {class }}$ of a randomly chosen joint is

$p_{f}=\frac{1}{\sigma} \int_{-\infty}^{\infty} \Phi(-\beta) \varphi\left(\frac{\beta-\mu}{\sigma}\right) d \beta \simeq 1.25 \cdot 10^{-5}, \beta_{\text {class }}=-\Phi^{-1}\left(p_{f}\right)=4.21<E[\beta]=4.40$ 


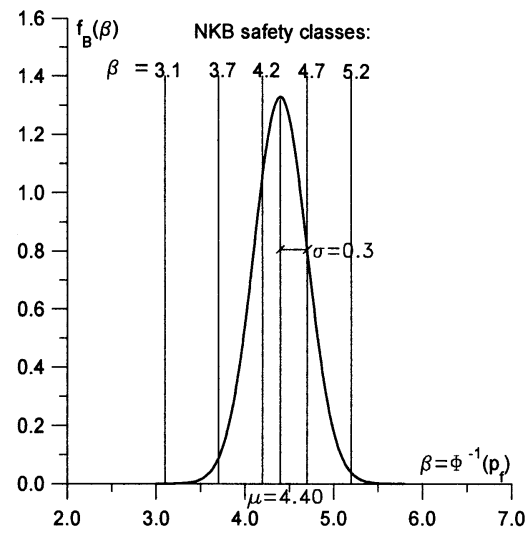

Fig. 1. Distribution of the reliability index as obtained by simulating deterministic designs of tubular joints according to a presently valid partial safety factor code for offshore structures (DS 449) when compared to a specific probabilistic code for the joint resistance and the external loads.

The problem is now whether the optimal target reliability index $\beta_{t}$ should be chosen as $\beta_{\text {class }}$ or whether it should be some other value of $\beta$. One argument could be that since the authority has accepted that the fraction $x$ of the joints according to the present code has a reliability index smaller than $\beta(x)=\mu+\sigma \Phi^{-1}(x)$, then $\beta(x)$ should in principle be acceptable as target reliability index if $x$ is not too small ( $x=25 \%$, for example). However, the problem is not solved before some rational principle of choosing $x$ is established.

\section{Optimal reliability}

The discussion of the problem about the choice of the reliability level can conveniently be supported on the following often considered simplified decision theoretical model.

The anticipated capital investment in a structure under design is obviously a function $c(p)$ of the required failure probability $p$. This function can be assumed to be decreasing. Assume that the loss of the structure by a failure will imply an investment equal to $c(p)+d$ where $d$ is the direct damage cost of the failure event (compensations for losses, clearing, etc.) measured in monetary units. For each value of $p$ we thus have a lottery (a game) between two consequences, namely a consequence with the utility $-c(p)$ corresponding to the event of no failure, and a consequence with the utility $-2 c(p)-d$ corresponding to the event of failure. These two consequences refer to a specific period of time. In order not to complicate the discussion with considerations on capitalizations (interest rate considerations) that require an extension of the model to keep account of the point in time at which the failure possibly occurs, the reference period is taken to be short like 1 year, say. The total cost then is

$c(p)+[c(p)+d] \mathbf{1}_{\text {failure }}, \quad \mathbf{1}_{\text {failure }}= \begin{cases}1 & \text { if failure occurs } \\ 0 & \text { otherwise }\end{cases}$ 
and it has the expected value $c(p)+[c(p)+d] p$. Following von Neumann's and Morgenstern's decision rule [9] we should among these infinitely many lotteries between two consequences choose the lottery for which the expected total cost is smallest. By differentiation we get the condition $c^{\prime}(p)(1+p)+c(p)+d=0$ for the determination of the optimal failure probability. For real structures the value of $d$ will generally be sufficiently large for $p$ to be very small. Assuming that $p<<1$ the last equation reduces to

$d \simeq-c(p)-c^{\prime}(p)$

from which $d$ is directly obtained as a function of $p$, or from which $p$ can be determined as a function of $d$.

The variation of the capital investment within the relevant interval of small failure probabilities can in many cases be approximated well by a function of $p$ which is linear in the generalized reliability index $c(p)-c\left(p_{0}\right)=a\left(\beta-\beta_{0}\right)$, where $a$ is the increase of cost per unit increase of $\beta$, and $\beta_{0}$ is a suitable representative reliability index value (e.g., $\beta_{0}=4.76$ corresponding to the probability $\left.p_{0}=\Phi\left(-\beta_{0}\right) \simeq 10^{-6}\right)$. The equation (5) then becomes

$\frac{d+c\left(p_{0}\right)}{a} \simeq \sqrt{2 \pi} e^{\frac{1}{2} \beta^{2}}-\left(\beta-\beta_{0}\right) \simeq \sqrt{2 \pi} e^{\frac{1}{2} \beta^{2}} \simeq \begin{cases}2.3 \cdot 10^{2} & \text { for } \beta=3 \\ 0.75 \cdot 10^{4} & \text { for } \beta=4 \\ 0.67 \cdot 10^{6} & \text { for } \beta=5\end{cases}$

Since $c\left(p_{0}\right) / a$ presumably is of order of size as $1, c\left(p_{0}\right)$ can obviously be neglected in the ratio $\left[d+c\left(p_{0}\right)\right] / a$ on the left side of $(6)$. In order to get optimal reliabilities of the size required in present codes the direct damage costs therefore must be several orders of magnitude larger than the investment $c(p)$.

For given $d$ and for values of $\beta$ that are relevant in structural reliability we thus find from (6) that the optimal reliability index is approximately

$\beta_{o p t} \simeq \sqrt{2 \log \left(\frac{d}{a \sqrt{2 \pi}}\right)}$

For an optimal value of $\beta$ equal to 4.5 a change of the ratio $d / a$ by a factor of 10 will imply a change of the optimal value of $\beta$ by about $10 \%$.

Let us as a vision assume that engineering practice for the choice of the target reliability in structural design develops in the direction of determination of the optimal reliability according to decision theoretical principles. Then the engineering profession must formulate regulating codes for certain utility value assessments that ensure a generally acceptable and authoritatively approved preference ordering of the consequences of any building activity. Codes for utility value specifications should in an initial phase be calibrated to such values that the code by and large leads to the reliability levels approved by the superior authority and pointed out by this authority on the basis of the existing codes. As shown this calibration will lead to very large values of the direct failure costs $d$. 
Often $d$ will be orders of magnitude larger than the compensations ordinarily paid out by the insurance companies. That the values of $d$ are so large may be seen as a consequence of the natural aversion of the engineering profession against experiencing failures. Such aversion can partly be due to a fear of loosing prestige and goodwill in the society but may also be seen as an ethical attitude that command the engineering activity to be practiced such that it will cause no harm to human health and life.

A practical consequence of the high reliability levels is that the serviceability requirements to the structures in many cases need not be checked. Thus the motive for the high reliability levels may be to free the designer for problems about the serviceability requirements. Definitely this motive has nothing ethical to it but it might be defendable from a cost benefit point of view.

If the optimality postulate stated by the superior authority is accepted, that is, if it is taken for grated that the reliability levels defined implicitely for certain structure type classes by a current authorized code is optimal [1] it turns out that the damage costs $d$ do not vary proportionally with realistic monetary compensation values. Thus the damage cost $d$ is a socio-economic value.

Besides specifying critical utility values in suitable units that can be transformed into monetary units a decision theoretical code just like a probabilistic code with specified target reliability levels should standardize a set of probability distribution types for the input variables.

\section{Uncertain gain and utility functions in the decision analysis}

Construction activities imply direct monetary costs that at the state of the design decision making are not known with certainty. The costs are assessed by considering present price levels and perhaps by setting up prognosis models for the future economical environments of the project. Such considerations may lead to an assessment of a probability distribution of the actual monetary costs. With respect to monetary costs the realization of the project is thus considered as a draw from this distribution, that is, as a run of a lottery. This lottery is a component of a composite lottery in the set of lotteries of relevance for the actual decision problem. When taking the expected value of the utility of the composite lottery the monetary cost component will contribute merely with its expected value. Thus it follows that the future monetary costs need only be assessed up to the expected value.

The decision maker may also be uncertain about his or hers preferences. This is reflected in uncertainty about the choice of the utility of the consequences $K$, that is, of the utility function $u(K)$. In the particular case where the utility is identified with the gain, the problem has been clarified above. If the considered consequence $K$ is not directly measurable on a monetary scale implying that a socio-economic utility value must be assiged to $K$, the problem may be dealt with in the same way. The uncertainty in the mind of the decision maker is expressed by his or hers assessment of a probability distribution of utility values rather than by a choice of a unique value. Thus the consequence $K$ is with respect to utility dissolved into a set of socio-economic values. A lottery between 
these values is included as a component of the relevant composite lottery. The lottery probabilities are given by the assessed probability distribution. As for the monetary gain it follows that it is sufficient to use the expected value in the utility distribution as the input utility value of the consequence $K$ in the decision model.

The simple conclusion of these considerations is that if von Neumann's and Morgenstern's decision axioms are accepted [9], then it should also be accepted that in presence of uncertain gain or utility functions it is only the expected gain or utility function which is relevant for the final decision problem.

As an example consider the variability of the reliability index of the tubular joints obtained by partial safety factor design as reported in Section 2 . This variability can be transformed into variability of $d$ by considering the ratio $d /(a \sqrt{2 \pi})$ obtained from (6) as a random variable $D /(a \sqrt{2 \pi})$. Thus the partial safety factor code as applied to the tubular joint class of structures gives uncertain information about the damage cost $d$. If all the variability is taken as an expression of uncertainty in the assessment of the value of $d$ (or better expressed, perhaps, the uncertainty left over by the superior authority to the code committee that faces the problem of calibrating a probabilistic code to an existing partial safety factor code), it follows from the decision theory that $d$ should be put to the expectation $E[D]$. Since

$\frac{E[D]}{a \sqrt{2 \pi}} \simeq \frac{1}{\sigma} \int_{-\infty}^{\infty} e^{\frac{1}{2} x^{2}} \varphi\left(\frac{x-\mu}{\sigma}\right) d x=\frac{1}{\sqrt{1-\sigma^{2}}} \exp \left[\frac{\mu^{2}}{2\left(1-\sigma^{2}\right)}\right]$

in which $\mu=4.40$ and $\sigma=0.30$, the optimal reliability index according to (7) becomes

$\beta_{\text {opt }} \simeq \sqrt{\frac{\mu^{2}}{1-\sigma^{2}}-\log \left(1-\sigma^{2}\right)} \simeq 4.62>E[\beta]$

This value is about as much larger than the expectation $E[\beta]=4.40$ as $\beta_{\text {class }}=4.21$ is smaller than the expectation, (3). It is seen that $E[D]$ increases with $\sigma$ and that $E[D] \rightarrow \infty\left(\beta_{\text {opt }} \rightarrow \infty\right)$ as $\sigma \rightarrow 1$. Of course, these specific conclusions can only be claimed to be valid for the example considered here, where the uncertainty distribution of $\beta$ is normal.

More refined analyses may be made in specific realistic examples of practice. For example, the coefficient $a$ in the expansion $c(p)-c\left(p_{0}\right)=a\left(\beta-\beta_{0}\right)$ can be a random variable when considering the variation over structures.

Remark If $\beta_{\text {opt }}$ as given by (9) is taken as the target reliability index $\beta_{t}$ for calibration of a design value code format the penalty function on the reliability index deviations from the target reliability index should ideally be such that it preserves the damage cost expectation $E[D]$. This suggests that the penalty function should be chosen such that it is a function of $\beta$ solely through $\exp \left[\frac{1}{2}\left(\beta^{2}-\beta_{t}^{2}\right)\right]-1$ (and not through $\beta-\beta_{t}$ as it is most often suggested). 


\section{Probabilistic code ambiguity}

For simplicity of analysis in this section consider a structure type for which a resistance $R$ and a load effect $S$ can be defined as two mutually independent random variable. The failure event is then the event $R-S<0$.

Let a structure with small uncertainty of the resistance $R$ relative to the uncertainty of the load effect $S$ be designed to the limit according to a current partial safety factor code. The result is a structure with given resistance $R$. Assume now that we are asked to formulate a probabilistic code that leads to exactly the same resistance $R$ of the considered structure. To do that we first need to specify the distribution types on which the probabilistic code should be based.

The examples in the appendix suggest three different probabilistic code formats, in the following denoted as Code $(\mathrm{N}, \mathrm{N})$, Code $(\mathrm{LN}, \mathrm{N})$, and Code $(\mathrm{LN}, \mathrm{LN})$ where $\mathrm{N} \sim$ normal distribution and $\mathrm{LN} \sim$ lognormal distribution. Code $(\mathrm{N}, \mathrm{N})$ is the format where both $R$ and $S$ are assigned normal distributions. This format is inconvenient except if the probability $P(R<0)$ is negligible as compared to the failure probability $P(R<S)$. In fact, Code $(\mathrm{N}, \mathrm{N})$ can by elementary calculations be shown to give

$\frac{E[R]}{E[S]}=\frac{1+\beta \sqrt{V_{R}^{2}+V_{S}^{2}-\beta^{2} V_{R}^{2} V_{S}^{2}}}{1-\beta^{2} V_{R}^{2}}$

which for $V_{R}=0$ is the same as (A.2). It is seen that $E[R] \rightarrow \infty$ as $V_{R} \rightarrow 1 / \beta$, which is an unreasonable behavior caused by the erraneous assignment of positive probability to the event $R<0$.

Ruling out Code $(\mathrm{N}, \mathrm{N})$ we are left with the two other formats: Code $(\mathrm{LN}, \mathrm{N})$ and Code (LN,LN). Since Code (LN,N) and Code $(\mathrm{N}, \mathrm{N})$ coincides in the limit $V_{R} \rightarrow 0$ we may calibrate Code (LN,N) by use of (A.2). To give the same design as determined by the partial safety factor code we thus should put $\beta$ in (A.2) to

$\beta_{N}=\left(\frac{R}{E[S]}-1\right) \frac{1}{V_{S}}$

However, for Code (LN,LN) we obviously should calibrate by use of (A.6) with $V_{R}=0$. Thus $\beta$ in (A.6) should be put to

$\beta_{L N}=\frac{1}{\sqrt{\log \left(1+V_{S}^{2}\right)}} \log \left[\frac{R}{E[S]} \sqrt{1+V_{S}^{2}}\right]$

It is seen that $\beta_{L N} \rightarrow \beta_{N}$ asymptotically as $V_{S} \rightarrow 0$. Otherwise $\beta_{L N}<\beta_{N}$. By substituting $R / E[S]$ from (11) into (12) we get the relation

$\beta_{L N}=\frac{\log \left[\left(1+\beta_{N} V_{S}\right) \sqrt{1+V_{S}^{2}}\right]}{\sqrt{\log \left(1+V_{S}^{2}\right)}}$ 
In case $V_{R}>0$ the relation between $\beta_{N}$ and $\beta_{L N}$ cannot be given in explicit analytical form. Fig. 2 shows the relation for different values of $V_{R}$ and $V_{S}$. For $V_{R}>0$ it is calculated approximatively by a standard first order reliability analysis method (FORM, Section 7) using the DNV program PROBAN.
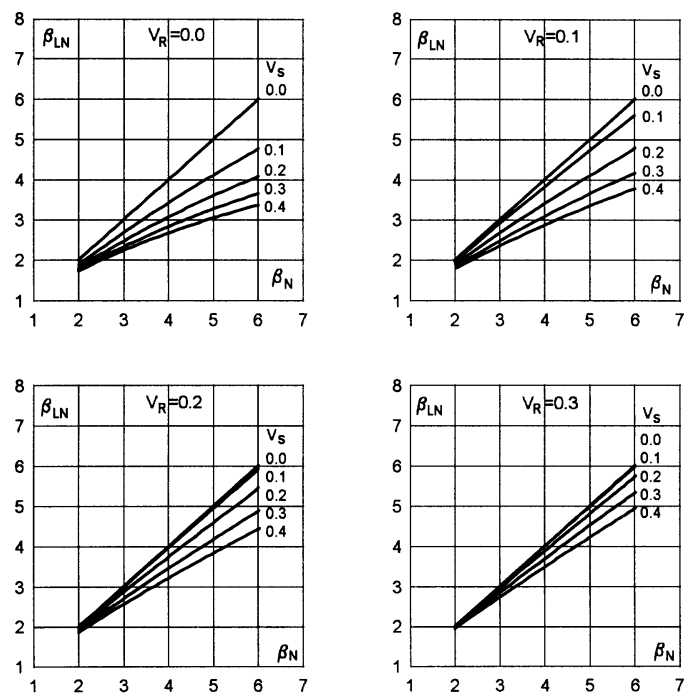

Fig. 2. Equivalent reliability indices $\beta_{N}$ and $\beta_{L N}$ corresponding to Code $(L N, N)$ and Code $(L N, L N)$, respectively.

It is seen that design with respect to constant reliability index of the one code implies a substantial variation of the reliability index of the other code. This is just an illustration of the old tail-sensitivity problem that since the early days of structural reliability theory repeatedly has been an obstacle of concern but also by many contributors to the field has been conveniently neglected or swept under the carpet. Under the optimality postulate the variation of the reliability index is by (6) directly transformed into a very large variation of the damage cost $d$. This emphasizes the role of $d$ as a formal calibration quantity that cannot be separated from the considered probabilistic reliability model. Also the large variability emphasizes the necessity of codifying a specific probabilistic reliability model as a standard reference code.

The two probabilistic code formats compared here are grossly simplified and serve only as an illustration of the code definition problem. Realistic probabilistic code formats will specify a set of different basic input variables that define geometrical quantities, material resistances and loads. Each code format specifies the joint probability distribution of the basic variables. The simple $(R, S)$ examples discussed here show the importance of making similar comparisons between different suggested realistic probabilistic code formats, and to choose one of these code formats as the basis for the standard reference code that 
should be calibrated to the optimal existing design practice as declared to be so by the superior authority.

\section{Prudency principle for choice of probabilistic code}

A principle of prudency can be applied to guide the choice of the standard reference probabilistic code format. Clearly it is a reasonable principle first to rule out all those code formats that specify distribution types that are strongly inconsistent with available empirical distributions and general engineering knowledge. The choice among the remaining code formats is arbitrary, as pointed out long ago by Freudenthal [10]. A requirement of reasonable calculational operability may rule out some further formats.

Left over with a finite set of different probabilistic code formats we need a principle for making a unique choice among these formats. Referring first to the tubular joint example with the limit state (1) the prudency principle is to choose that code format for which there is minimum of the coefficient of variation of the reliability index for the population of designs obtained according to the given partial safety factor code with fixed factor values. Thus the prudency principle advocates an attitude of minimal change of current design practice.

This prudency principle may lead to different probabilistic code formats in dependence of the considered structure type class with associated type of ultimate limit state. To overcome this ambiguity problem the coefficient of variation of the reliability index can be treated as a random variable defined on the population of relevant structure types. The prudency principle may then be to choose that probabilistic code format for which the expectation of the coefficient of variation is minimal. To be operational, this principle requires that probabilities are assigned to the different considered ultimate limit states. These probabilities could reasonably be chosen to reflect the relative frequency of occurrence of the structure type in practice.

For each structure type with associated ultimate limit state there is an infinity of realizations of the limit state surface. In the tubular joint example the population of these realizations was defined by randomly generating the influence coefficients by which the external loads are transformed into the internal forces of the joint. Clearly this way of obtaining the population of limit state surfaces is used in the example just for illustration. A realistic code calibration excercise should be based on countings and structural analysis investigations to assess the relative frequency of occurrence of the different influence coefficients in $\boldsymbol{C}$.

\section{$7 \quad$ Partial safety factor code calibration}

After the choice of a reference probabilistic code format a specific probabilistic code should be established through assignment of parameter values, certain cost values (intangibles), and/or reliability levels such that the code at the time of its authorization possesses socio-economic optimality as assessed by the superior authority. The probabilistic code may then be used directly in engineering practice. However, for the design of standard 
types of structures it may be more convenient and operational to base the routine design work on a partial safety factor code format. Therefore it is relevant to determine partial safety factor values by calibration to designs obtained by use of the reference probabilistic code. It goes without saying that this partial safety factor code only approximates the probabilistic code reasonably well if it is allowed to contain several sets of partial safety factor values to be used in dependence of structure type and ultimate limit state type.

To appreciate this statement it is illustrative to study the relation between the partial safety factor concept and the reliability index concept. To make the text directly accessible to non-specialists of probabilistic reliability calculus the standard approximative procedure known as FORM (First Order Reliability Method) is explained here in its simplest possible form.

Consider a reliability problem with only two random variables $X_{1}$ and $X_{2}$. Let the limit state be a curve in the $\left(x_{1}, x_{2}\right)$-plane defined by the equation $g\left(x_{1}, x_{2}\right)=0$, where $g\left(x_{1}, x_{2}\right)$ is a function defined on the basis of structural analysis or experiments. Moreover assume that $X_{1}$ and $X_{2}$ are mutually independent random variables with distribution functions $F_{X_{1}}(x)$ and $F_{X_{2}}(x)$ (三 the probability that $X_{2} \leq x$, an input information), respectively. Then define the mapping

$u_{1}=\Phi^{-1}\left[F_{X_{1}}\left(x_{1}\right)\right], \quad u_{2}=\Phi^{-1}\left[F_{X_{2}}\left(x_{2}\right)\right]$

of the $\left(x_{1}, x_{2}\right)$-plane onto the $\left(u_{1}, u_{2}\right)$-plane, where $\Phi^{-1}(\cdot)$ is the inverse function to the standard normal distribution function $\Phi(\cdot)$. This mapping has the property that the corresponding random variable pair $\left(U_{1}, U_{2}\right)$ obtained from $\left(X_{1}, X_{2}\right)$ has the rotational symmetric 2-dimensional standard normal density function

$f_{U_{1}, U_{2}}\left(u_{1}, u_{2}\right)=\varphi\left(u_{1}\right) \varphi\left(u_{2}\right)=\frac{1}{2 \pi} \exp \left[-\frac{1}{2} r^{2}\right]$

where $r^{2}=u_{1}^{2}+u_{2}^{2}$. In the $\left(u_{1}, u_{2}\right)$-plane the limit state becomes represented by a curve defined by the equation

$g\left(F_{X_{1}}^{-1}\left[\Phi\left(u_{1}\right)\right], F_{X_{2}}^{-1}\left[\Phi\left(u_{2}\right)\right]\right)=0$

For any reasonable reliability problem this curve divides the $\left(u_{1}, u_{2}\right)$-plane in two parts such that the origin is in the image part of the safe set in the $\left(x_{1}, x_{2}\right)$-plane. Thus the probability $p_{f}$ of the failure event is the same as the probability obtained from the normal density (15) integrated up over that part of the $\left(u_{1}, u_{2}\right)$-plane that does not contain the origin. For simplicity assume that the curve (16) is a straight line. Then the rotational symmetry of the normal density about the origin ensures that $p_{f}$ is determined solely by the distance $\beta$ from the origin to the straight line. In fact, we have $p_{f}=\Phi(-\beta)$ or $\beta=-\Phi^{-1}\left(p_{f}\right)$.

Even though the limit state curve in the $\left(u_{1}, u_{2}\right)$-plane is rarely a straight line the curvature at the point closest to the origin is most often so small that the curve for reliability evaluation purposes can be replaced by the tangent at this point. Thus the geometric reliability index (Hasofer-Lind index [11]) defined as the smallest distance from 
the origin to the limit state curve is most often a very good approximation to the reliability index defined by $\beta=-\Phi^{-1}\left(p_{f}\right)$.

The situation is illustrated in Fig. 3 with two different limit states 1 and 2 . The radius $\beta$ of the circle defines the common reliability level. If this $\beta$ is the target reliability level the value of the design variable (reinforcement area, say) in the limit state problem 1 is determined such that the limit state curve 1 becomes tangential to the circle. In its most elementary form this is design according to the reliability index method. For this particular design there are an infinity of partial safety factor pairs applied to given characteristic values for $X_{1}$ and $X_{2}$. Clearly all what is needed is arbitrarily to define a single design point $Q$ on the limit state curve, given that the family of limit state curves for the design situation 1 is a one-parameter family with the design variable as parameter. If $D_{1}$ is chosen as design point, where $D_{1}$ is the point on the limit state curve 1 closest to the origin $\left(D_{1}\right.$ is in the literature conveniently called the "most central" or "most likely" limit state point, but less conveniently it also appears with the name "design point"), the partial safety factors that produce the same design become

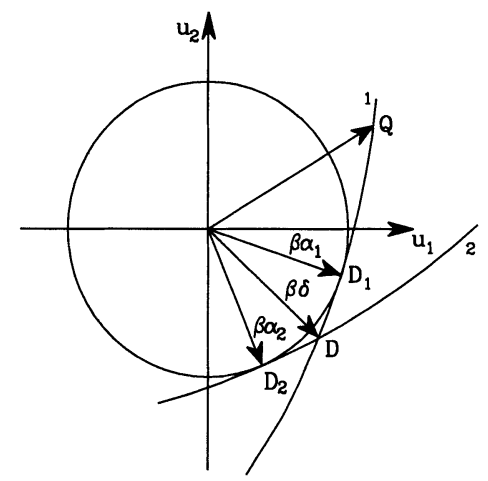

Fig. 3. Illustration of design points corresponding to a given geometric reliability index $\beta$ (Q: ambiguous for one limit state; $D$ : unique for two limit states, single design points non-existent for more than two limit states).

$\gamma_{11}=\frac{F_{X_{1}}^{-1}\left[\Phi\left(\beta \alpha_{11}\right)\right]}{x_{1 k}}, \quad \gamma_{12}=\frac{x_{2 k}}{F_{X_{2}}^{-1}\left[\Phi\left(\beta \alpha_{12}\right)\right]}$

where (in accordance with Fig. 3) $x_{1 k}$ is an upper fractile characteristic value of $X_{1}$ (load type variable) and $x_{2 k}$ is a lower fractile characteristic value of $X_{2}$ (resistance type variable), e.g. $x_{1 k}=F_{X_{1}}^{-1}\left(p_{1}\right), x_{2 k}=F_{X_{2}}^{-1}\left(p_{2}\right)$ with $p_{1}=0.98$, say, and $p_{2}=0.05$, say. For the other limit state 2 the point $D_{2}$ may be taken as design point and partial safety factors $\gamma_{21}, \gamma_{22}$ be calculated.

Needless to say, since the partial safety factor method only serves a purpose if it can be used directly for design without first making a design according to the reliability index method, it is necessary that the partial safety factors be fixed at least within a reasonably wide class of design problems. For the case with the two limit states in Fig. 3 
it is immediately seen that if the intersection point $D$ between the two limit state curves is taken as design point, then the partial safety factors are common for the two limit states. The vector $\boldsymbol{\delta}$ acts as a replacement vector for $\boldsymbol{\alpha}_{1}$ and $\boldsymbol{\alpha}_{2}$ without introducing any error of approximation. Clearly, with more than two limit states in the class of design problems containing solely the two random variables $X_{1}$ and $X_{2}$, there is no replacement vector $\boldsymbol{\delta}$ that exactly reproduces all the designs. By a given superior requirement there will only be allowed one design point $\beta \boldsymbol{\delta}$ for the class, and all the limit state curves are therefore adjusted to contain this point. Thus the reliability indices will vary over the class. The strategy is then to determine the replacement vector $\boldsymbol{\delta}$ such that the expected loss (penalty) for the entire design class becomes minimal, [12]. Of course, the range of obtained reliability index values within the class should be controlled. If the range is judged to be too wide, the class should be divided into subclasses each with its own set of partial safety factors.

In this connection it is of interest to evaluate the sensitivity of the replacement vector $\boldsymbol{\delta}$ and the corresponding partial safety factors to changes of parameters. The parameters may have influence on the limit state before the mapping into the standard Gaussian space (the $\left(u_{1}, u_{2}\right)$-plane) or they may have influence on the probability distributions of the basic variables. If the reliability index is kept at the target value $\beta$ all what essentially happens in the example of Fig. 3 by a minor parameter variation is that each limit state curve is changed to another curve close by the first curve. This new curve is also tangential to the circle of radius $\beta$. The averaging effect of the penalty optimization will tend to cause that the change of the replacement vector $\delta$ will be smaller than the changes of the individual $\boldsymbol{\alpha}$-vectors.
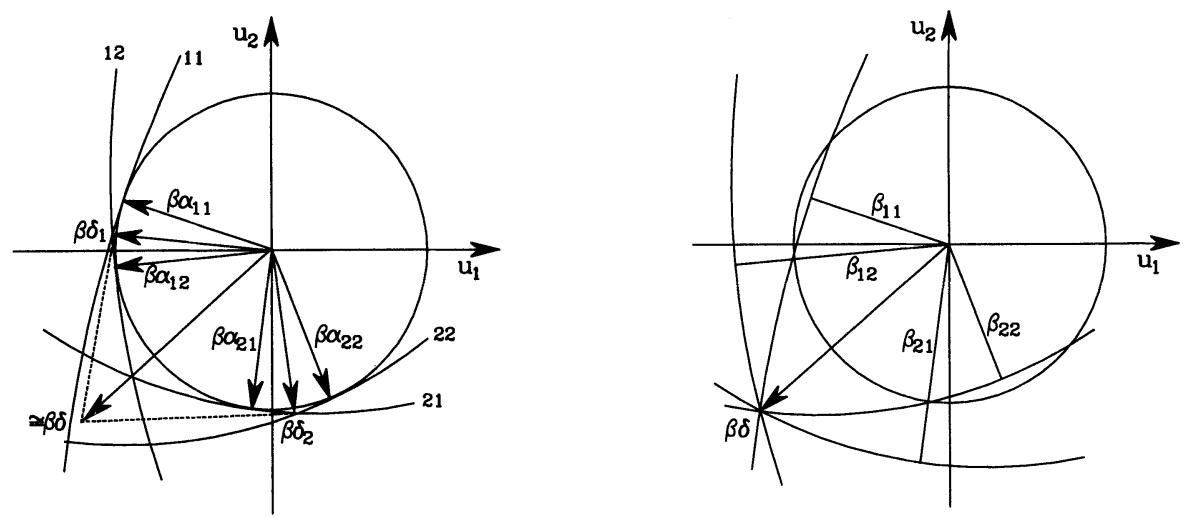

Fig. 4. Illustration of two clusters of most central limit state points separated by an angle of about $90^{\circ}$ with well approximating separaie replacement vectors $\boldsymbol{\delta}_{1}, \boldsymbol{\delta}_{2}$ and the resulting less good approximating common replacement vector $\boldsymbol{\delta}$.

There is a particularly important issue related to the classification of design problems into classes of constant partial safety factors. For limit states of the same mechanical type (same collapse mode) the $\boldsymbol{\alpha}$-vectors as defined in Fig. 3 tend to be close to each other 
such that the corresponding most central points form a cluster on the sphere of radius $\beta$ (in Fig. 3 the points $D_{1}$ and $D_{2}$ on the circle of radius $\beta$ ). As an example consider reinforced concrete, and in particular consider as one type the bending failure of a beam cross-section where the yield moment is mainly determined by the yield strength $X_{1}$ of the tension reinforcement and, as another type, the compression failure of a short column where the carrying capacity is mainly determined by the compression strength $X_{2}$ of the concrete. Then the situation in the $\left(u_{1}, u_{2}\right)$-plane is as scetched in Fig. 4. For the bending failure limit states the variability of $X_{2}$ has only small influence and the cluster of most central limit state points is therefore situated on the circle in the vicinity of $(-\beta, 0)$. For the limit states of compression failure type the cluster of most central limit state points is similarly situated on the circle in the vicinity of $(0,-\beta)$, about $90^{\circ}$ apart from the first cluster of points. If the two limit state types are put in two different design case classes we get the two optimal replacement vectors $\boldsymbol{\delta}_{1}$ and $\boldsymbol{\delta}_{2}$ centrally placed within each cluster, as illustrated in Fig. 4 (left). If both types of limit states are required to be treated by the same partial safety factors, that is, if they are put together in one class, the optimal replacement vector $\boldsymbol{\delta}$ will have some direction between the two clusters depending on the relative weighting of the two types. Fig. 4 shows two limit states of each type. With equal weight on the two types a first crude assessment of an optimal $\delta$ is obtained by letting $\beta \delta$ be the vector to the crossing points of the two orthogonals to $\delta_{1}$ and $\boldsymbol{\delta}_{2}$ at the points $\beta \boldsymbol{\delta}_{1}$ and $\beta \boldsymbol{\delta}_{2}$, respectively.

Fig. 4 (right) illustrates the possible consequence of having a single replacement vector $\boldsymbol{\delta}$ for the $\boldsymbol{\alpha}$-vectors of about $90^{\circ}$ separated clusters of most central limit state points. All the limit states are now required to contain the point $\beta \delta$. Obviously the approximation errors for the reliability indices increase when extending the class of design cases. What intuitively can be seen from Fig. 5 is that from having quite small approximation errors when keeping $\boldsymbol{\delta}_{1}$ and $\boldsymbol{\delta}_{2}$ for practical design, the error may blow up to considerable size when requiring that there should only be one set of partial safety factors.

Whether the minor complication of having some few classes of partial safety factors is of sufficient inconvenience to justify the resulting much larger reliability variability when there is only a single class, can in principle be decided in a rational way by a cost benefit analysis. At the very least it must be ensured that there within the class is no relevant structure for which the reliability index becomes much smaller than the target reliability index.

\section{Conclusions}

Some of the following conclusions are about facts while other conclusions are of a debatable philosophical or political nature expressing the opinion of the author. The author sees that there is an increasing need for establishing standard reference probabilistic code formats. Therefore a debate in the profession about this issue would be wellcome. The author's conclusions are:

1. The reliability index of a limit state formulated for a given structure carries information about the structural reliability with respect to the limit state only if the reliability index 
is reported together with a reference to the specific probabilistic code format by which it is defined. Constant reliability in one code does not imply constant reliability in another code.

2. A specific probabilistic code format should be declared to be the authorized reference code for constant reliability.

3. The choice of the authorized probabilistic reference code format can be based on a principle of prudency after ruling out those formats that are significantly inconsistent with available generally valid empirical data and scientific evidence. By the principle of prudency that probabilistic format is chosen by which the reliability index variation as induced by a current authorized partial safety factor code in a certain sense becomes as small as possible.

4. A partial safety factor code with a fixed set of safety factor values induces a considerable variation of any reliability index over the set of structures designed to the limit according to the safety factor code.

5. Under the postulate that the intention of any code is that it should produce designs among which there are at least some designs that are optimal in a socio-economic sense, the reliability index uncertainty induced by the code can be transferred into damage cost uncertainty. The optimal designs should be pointed out by a superior authority.

6. The damage cost distribution induced by a given authorized code can for the calibration of a probabilistic code to the given code be taken as an expression of uncertainty about the damage cost assessment. Adopting the decision theory (von Neumann and Morgenstern) as a superior basis for rational decision making the damage cost uncertainty distribution can simply be replaced by the expected value of the distribution. The corresponding reliability index value is then the calibration result.

\section{Acknowledgements}

Ph.D. students Claus F. Christensen, Johannes M. Johannesen, and Søren RandrupThomsen are acknowledged for computer programming help. The Joint Committee on Structural Safety has discussed the topic and its members are acknowledged for valuable and quite critical comments to the first draft of the paper. This work has been financially supported by the Danish Technical Research Council.

\section{References}

1. N.C. Lind, i) Reliability-based structural codes. Optimization theory. ii) Reliability-based structural codes. Practical calibration, iii) Safety level decisions and socio-economic optimization. In Safety of Structures under Dynamic Loading, Holland, Kavlie, Moe, Sigbjørnson, eds., Vol. 1, Tapir, Trondheim, 1978, 135-175.

2. J.D. Sørensen and I. Enevoldsen, Sensitivity weaknesses in application of some statistical 
distributions in First Order Reliability Methods, Structural Safety, 12 (1993) 315-325.

3. A.R. Flint, B.W. Smith, M.J. Baker, and W. Manners, Proc. Conf. on the New Code for the Design of Steel Bridges, Cardiff, 1980. Granada Publishing, 1981.

4. The Nordic Committee on Building Regulations (NKB), Recommendation for Loading- and Safety Regulations for Structural Design, NKB-Report No 36, Ministry of Housing, Copenhagen, 1978.

5. P. Thoft-Christensen and M.J. Baker, Structural Reliability Theory and its Applications. Springer Verlag, Berlin, Heidelberg, New York, 1982.

6. O. Ditlevsen and H.O. Madsen, Proposal for a code for the direct use of reliability methods in structural design. Working Document of the Joint Committee on Structural Safety (JCSS), IABSE-AIPC-IUBH, ETH-Hönggerberg, Zürich, 1989.

7. DS 449, Dansk Ingeniørforening's Code of Practice for Offshore Steel Structures on Pile Foundations (in Danish: Norm for pælefunderede offshore stålkonstruktioner), 1. edition. Teknisk Forlag, Copenhagen, 1983.

8. P. Bjerager, Offshore Konstruktioner 5947, Notat 8, Pålidelighedsanalyse. Teaching note in Danish. Department of Structural Engineering, Technical University of Denmark, 1985.

9. Von Neumann and Morgenstern, Theory of Games and Economical Behavior. Princeton University Press, 1943.

10. A. Freudenthal, Introductory Remarks, Proc. of ICOSSAR '72 (International Conference on Structural Safety and Reliability), A. Freudenthal, ed., Pergamon Press, Oxford, 1972, 5-6.

11. A.M. Hasofer and N.C. Lind, An exact and invariant first-order reliability format, Journal of Eng. Mech., ASCE, 100 (1974) 111-121.

12. K. Skov and R. Rackwitz, Note on the evaluation of partial safety factors for unknown and partially known shape of the limit state problem. DIALOG 77, Danish Engineering Academy, Lyngby, 1977, 99-115.

\section{APPENDIX}

As in the section on probabilistic code ambiguity consider a structure type for which resistance $R$ and load effect $S$ can be defined as two mutually independent random variables. The failure event is then the event that $R-S<0$.

Assume that the cost $c(p)$ within the domain of practical interest can be written as $c(p)=h+k E[R]$, where $h$ and $k$ are given constants while $R$ is the random resistance of the structure. Then (5) can be written as

$$
\begin{aligned}
& d+c\left(p_{0}\right) \simeq \\
& -\left[c(p)-c\left(p_{0}\right)\right]-\frac{d}{d p}\left[c(p)-c\left(p_{0}\right)\right]=k\left\{\left(E[R]-E\left[R_{0}\right]\right)+\frac{1}{\varphi(\beta)} \frac{d}{d \beta}\left(E[R]-R\left[R_{0}\right]\right)\right\}
\end{aligned}
$$

where $\beta_{0}=-\Phi^{-1}\left(p_{0}\right)$ is a suitable representative reliability index and $R_{0}$ is the random resistance when the structure is designed to have the reliability index $\beta_{0}$. 
First consider the simple example where the randomness of $R$ is so small that it can be neglected as compared to the randomness of the load effect $S$, and where $S$ has a Gaussian distribution. Then we simply have

$E[R]=E[S]\left(1+\beta V_{S}\right)$

where $V_{S}=D[S] / E[S]$ is the coefficient of variation of $S$. Thus (A.1) can be written as

$\frac{d+c\left(p_{0}\right)}{a} \simeq \frac{1}{\varphi(\beta)}-\left(\beta-\beta_{o}\right) \simeq \sqrt{2 \pi} e^{\beta^{2} / 2}$

where $a=k E[S] V_{S}$. Next consider the example where $R$ and $S$ both have lognormal distributions. Then

$E[\log R]=E[\log S]+\beta \sqrt{\operatorname{Var}[\log R]+\operatorname{Var}[\log S]}$

or

$\log E[R]-\frac{1}{2} \log \left(1+V_{R}^{2}\right)=\log E[S]-\frac{1}{2} \log \left(1+V_{S}^{2}\right)+\beta \sqrt{\log \left(1+V_{R}^{2}\right)+\log \left(1+V_{S}^{2}\right)}$

so that

$\frac{E[R]}{E[S]}=\sqrt{\frac{1+V_{R}^{2}}{1+V_{S}^{2}}} \exp \left\{\beta \sqrt{\log \left[\left(1+V_{R}^{2}\right)\left(1+V_{S}^{2}\right)\right]}\right\}$

Thus

$\frac{E[R]}{E\left[R_{0}\right]}=\exp \left\{\left(\beta-\beta_{0}\right) \sqrt{\log \left[\left(1+V_{R}^{2}\right)\left(1+V_{S}^{2}\right)\right]}\right\}$

and (A.1) reads

$\frac{d+c\left(p_{0}\right)}{a}=\frac{1}{b}\left\{\exp \left[b\left(\beta-\beta_{o}\right)\right]\left[1+\frac{b}{\varphi(\beta)}\right]-1\right\} \simeq \sqrt{2 \pi} e^{\beta^{2} / 2}$

where $a=k E\left[R_{0}\right] b$ and $b=\sqrt{\log \left[\left(1+V_{R}^{2}\right)\left(1+V_{S}^{2}\right)\right]}$. 